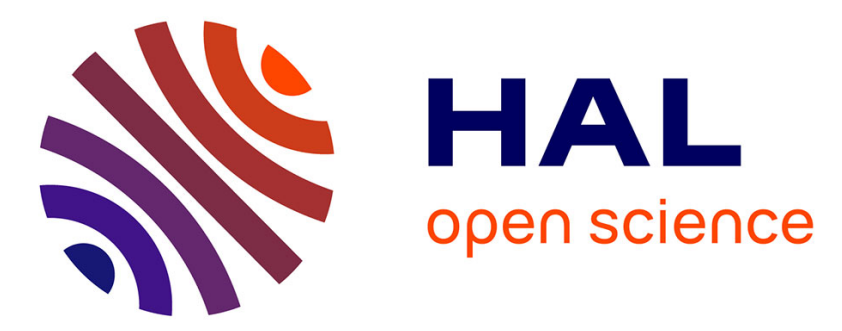

\title{
Terahertz metamolecules deposited on thin flexible polymer: design, fabrication and experimental characterization
}

Riad Yahiaoui, Keisuke Takano, Fumiaki Miyamaru, Masanori Hangyou, Patrick Mounaix

\section{To cite this version:}

Riad Yahiaoui, Keisuke Takano, Fumiaki Miyamaru, Masanori Hangyou, Patrick Mounaix. Terahertz metamolecules deposited on thin flexible polymer: design, fabrication and experimental characterization. Journal of Optics, 2014, 16 (9), pp.094014 (1-6). 10.1088/2040-8978/16/9/094014 . hal-01064121

\section{HAL Id: hal-01064121 \\ https://hal.science/hal-01064121}

Submitted on 19 Feb 2016

HAL is a multi-disciplinary open access archive for the deposit and dissemination of scientific research documents, whether they are published or not. The documents may come from teaching and research institutions in France or abroad, or from public or private research centers.
L'archive ouverte pluridisciplinaire HAL, est destinée au dépôt et à la diffusion de documents scientifiques de niveau recherche, publiés ou non, émanant des établissements d'enseignement et de recherche français ou étrangers, des laboratoires publics ou privés.

\section{(ㅇ)(1) $\$$}

Distributed under a Creative Commons Attribution - NonCommercial| 4.0 International 
Terahertz metamolecules deposited on thin flexible polymer: design, fabrication and experimental characterization

This content has been downloaded from IOPscience. Please scroll down to see the full text. 2014 J. Opt. 16094014

(http://iopscience. op.o g/2040-8986/16/9/094014)

View the table of contents for this issue, or go to the journal homepage for more

Download details:

IP Address: 147.210.24.83

This content was downloaded on 08/09/2014 at 10:08

Please note that terms and conditions apply. 


\title{
Terahertz metamolecules deposited on thin flexible polymer: design, fabrication and experimental characterization
}

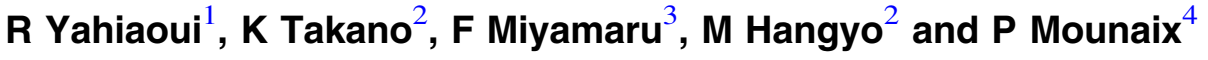 \\ ${ }^{1}$ XLIM, Univ. Limoges, CNRS, UMR 6172, 7 rue Jules Vallès, F 19100 Brive, France \\ ${ }^{2}$ Institute of Laser Engineering, Osaka University, 26 Yamadaoka, Suita, Osaka 565 0871, Japan \\ ${ }^{3}$ Department of Physics, Faculty of Science, Shinshu University, Nagano 390 8621, Japan \\ ${ }^{4}$ LOMA, Univ. Bordeaux, CNRS, UMR 5798, 351 Cours de la Libération, F 33405 Talence Cedex, France \\ E mail: riad.yahiaoui@unilim.fr
}

\begin{abstract}
Metamolecules deposited on thin dielectric film using standard optical lithography have been investigated numerically and experimentally in the terahertz regime. The topology of the proposed metamolecules is able to achieve a multiple band frequency response over a broad frequency range. The analysis of the spectral response of the investigated metamaterial reveals that such a feature arises from coupling effects between its individual constituents. The successful demonstration of a THz flexible metamaterial may open up new perspectives towards achieving complex electromagnetic functions involving non-planar metamaterials with simple design and fabrication.
\end{abstract}

Keywords: metamaterials, coupling mechanisms, terahertz time domain spectroscopy

\section{Introduction}

Metamaterials have grown substantially within the scientific community due to their unusual properties and their ability to control electromagnetic waves in an amazing manner $[1,2]$. Usually based on dielectric particles or metallodielectric composite structured on a size scale much smaller than the wavelength of external stimuli, they are increasingly used to achieve various electromagnetic functions such as absorption, radiation, filtering, coupling, amplification, super-resolution and so on. Their precise arrangement, geometrical dimensions and orientation can affect light in a way that is unexpected with conventional materials. The initial research in metamaterials dates back to the late 1960s with the early work of V G Veselago [3], where he theorized a new type of material that can exhibit negative electrical permittivity $(\varepsilon)$ and negative magnetic permeability $(\mu)$ simultaneously over a common frequency band, thus giving rise to negative refractive index $(n)$.

These materials, commonly called negative index materials (NIMs), remained for long time as 'science fiction' due to the unavailability of such materials in nature. Significant progress has been made through the work of J B Pendry et al, who proposed a composite structure exhibiting a negative refractive index. The structure, which combines plasmonic wires (PWs) [4] and split ring resonators (SRRs) [5], paved the way to the first experimental demonstration of the negative refraction in the microwave regime [6]. Later on, a tremendous variety of structures were proposed and contributed favourably to the considerable evolution of this new generation of materials [ 712 , , and the number continues to grow to this day.

The terahertz $(\mathrm{THz})$ regime, that extends from $100 \mathrm{GHz}$ to $10 \mathrm{THz}$, is a particularly interesting region, which remained inaccessible for long time due to the absence of appropriate 


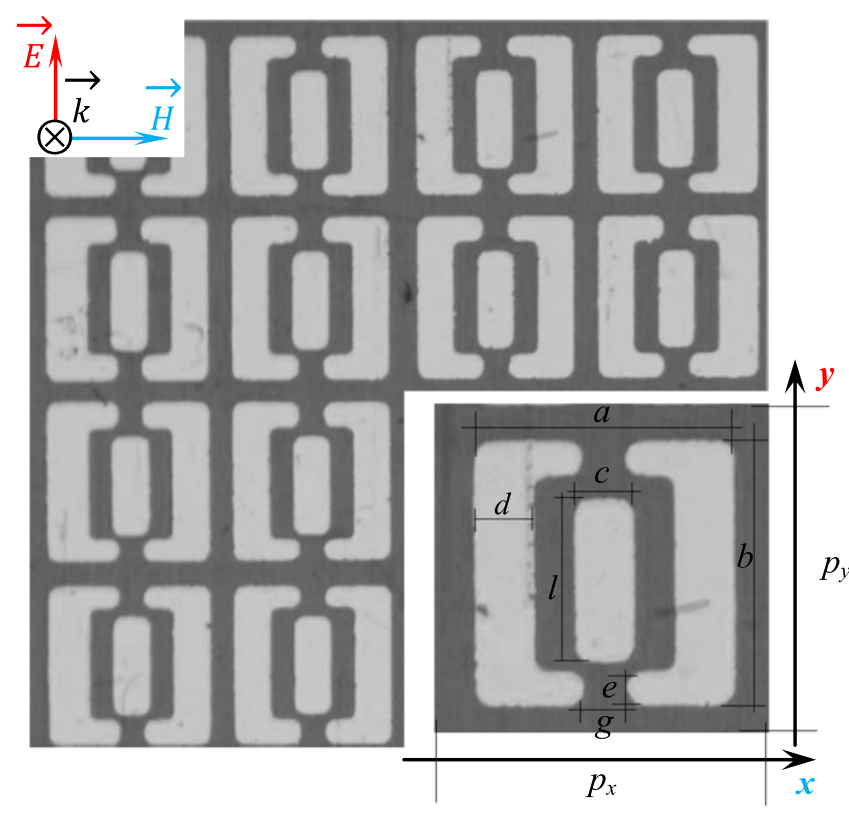

Figure 1. (a) Microscope image of the fabricated metamaterial with the relevant geometrical dimensions $p_{x}=p_{y}=300 \mu \mathrm{m}$, $a=b=250 \mu \mathrm{m}, c=d=50 \mu \mathrm{m}, e=20 \mu \mathrm{m}, g=50 \mu \mathrm{m}, l=150 \mu \mathrm{m}$, $t_{\text {substrate }}=50 \mu \mathrm{m}$.

emitters and detectors [13]. Recently, this frequency band experienced renewed interest due to advances in semiconductor technology, which gives rise to inexpensive sources and detectors, thus constituting a breakthrough for many useful applications, including subdiffraction imaging [14], cloaking [15], near perfect absorption [16, 17], polarization conversion [18, 19], sensing [20 23] etc.

In this work, we propose a metamaterial based on metamolecules supported by a flexible polymer operating in the terahertz regime. The electromagnetic behavior of the structure has been investigated numerically using the finite element method and experimentally by means of terahertz time domain spectroscopy (THz-TDS). Our results reveal that the metamolecules exhibit an electromagnetic behavior that alternates between rejection and transparency characteristics in the $0.11 \mathrm{THz}$ frequency range, potentially suitable for spatial filtering, antennas and sensing applications.

\section{Presentation of the investigated metamaterial}

The proposed metamaterial is shown in figure 1 . The elementary cell of the structure combines an inner cut wire $(\mathrm{CW})$ and an outer two-gap split ring resonator (SRR), made of $200 \mathrm{~nm}$ thick aluminum. They are printed on the top side of a $50 \mu \mathrm{m}$ thick Kapton ${ }^{\circledR}$ substrate using standard optical lithography, with the relevant geometrical dimensions $p_{x}=p_{y}=300 \mu \mathrm{m}, \quad a=b=250 \mu \mathrm{m}, \quad c=d=50 \mu \mathrm{m}, \quad e=20 \mu \mathrm{m}$, $g=50 \mu \mathrm{m}, l=150 \mu \mathrm{m}$. Considered as the fundamental building blocks of various metamaterials, the SRR and the CW have been widely used for constructing materials with negative permeability, negative permittivity and even negative refractive index when combined together [4 6]. The commercial dielectric substrate Kapton ${ }^{\circledR}$ that was considered in simulations and used in the fabrication process has a relative dielectric permittivity $\varepsilon_{\mathrm{r}} \sim 3$, and a moderate loss level ( $\tan \delta \sim 0.05$ ) up to $2 \mathrm{THz}$ (controlled by THz-TDS). Due to its small thickness, the chosen dielectric spacer has a very good mechanical flexibility, which is highly sought for applications with conformal geometries. The proposed metamaterial is obviously anisotropic and was illuminated by a TM-polarized incidence plane wave (i.e., $E \| \mathrm{CWs}$ ), with a single layer along the direction of propagation $k$. Note that an infinite structure was considered in the simulations along $E$ and $H$ fields, with a perfect conductor nature of the metallic constituents (SRRs and CWs), while a prototype covering a more realistic surface of about $2 \times 2 \mathrm{~cm}^{2}$ was fabricated.

\section{Numerical simulations and experimental measurements}

Numerical simulations based on the finite element method (FEM) were carried out by applying necessary periodic boundary conditions. The elementary cell of the designed metamaterial was placed inside an air box with lateral dimensions of $300 \mu \mathrm{m} \times 300 \mu \mathrm{m}$ and was illuminated by a normally incident plane wave with the electric field parallel to the $y$-axis and the magnetic field parallel to the $x$-axis. Perfect electric conductor (PEC) boundary conditions were applied along the $y$-axis, perfect magnetic conductor (PMC) boundary conditions were applied along the $x$-axis, and open boundary conditions were applied in the $z$-direction (direction of propagation $k$ ).

Measurements using THz-TDS were made in order to characterize the electromagnetic behavior of the structure. A schematic diagram and a photograph of the THz-TDS setup are shown in figure 2(a). Photoconductive (PC) antennas were used as the emitter and detector of the $\mathrm{THz}$ pulses. The excitation source of the PC antennas was a femtosecond fibre laser, whose centre wavelength, repetition rate and pulse width were $780 \mathrm{~nm}, 80 \mathrm{MHz}$ and $88 \mathrm{fs}$, respectively. The THz pulses were horizontally polarized and collimated by an offaxis parabolic mirror. The beam diameter was approximately $10 \mathrm{~mm}$ on the sample. In the transmission measurements, the incident plane waves are normal to the sample surface and the transmitted $\mathrm{THz}$ electric field is calibrated with respect to transmission in free space between the emitter and the receiver.

The simulated (solid line) and measured (hollow circles) frequency-dependent amplitude transmission of the metamaterial slab is shown in figure 2(b), with a very good agreement. One can observe a double transmission window around 0.46 and $0.89 \mathrm{THz}$. Parametric study performed on the designed metamaterial but not represented here has shown that the response of the metamaterial is determined by the size, the geometry of the elementary cell, the periodicity and the properties of the dielectric substrate.

In order to attempt to understand the origin of the transmission feature, we have further performed numerical simulations separately on the SRR only and CW only (the 

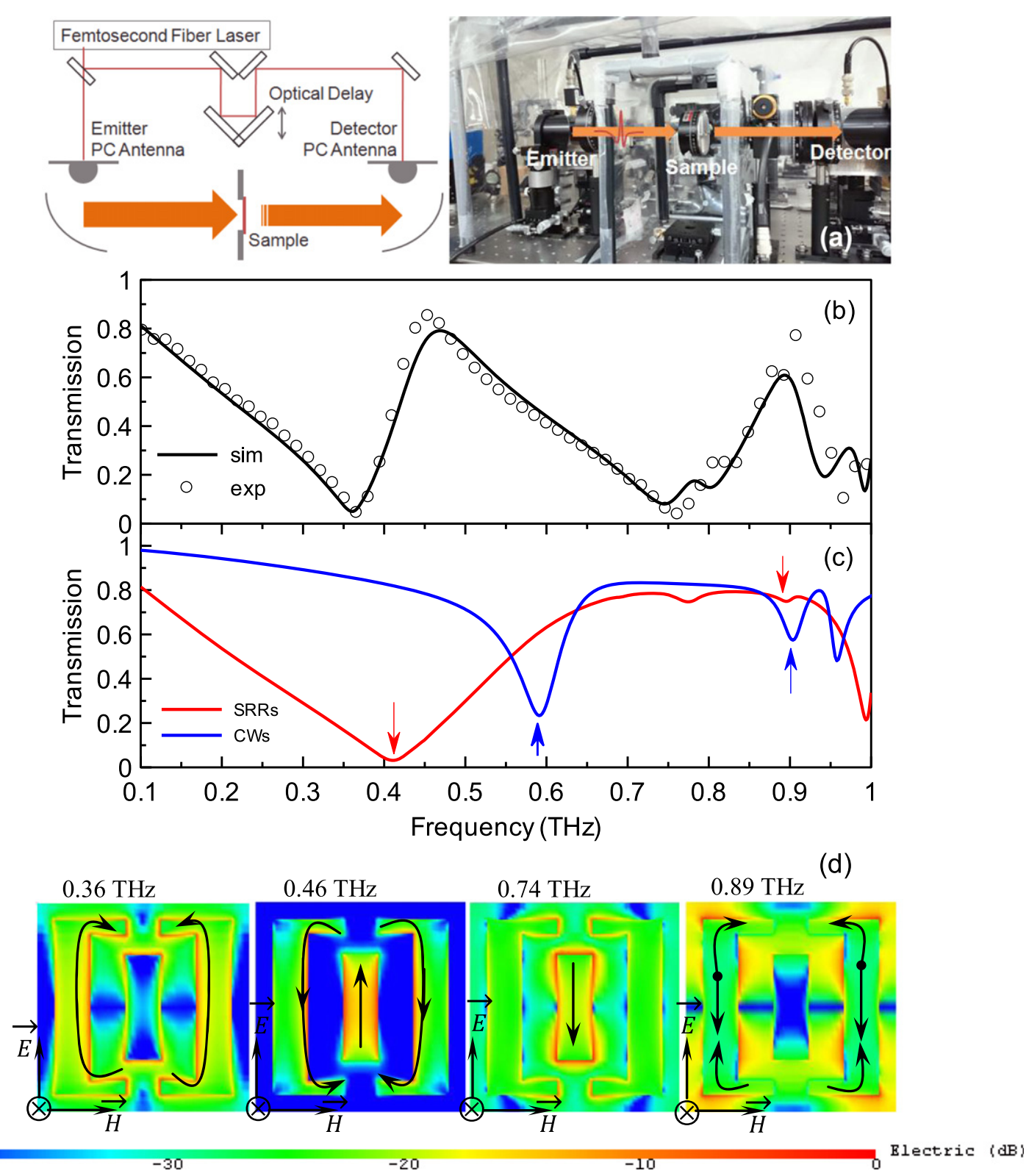

Figure 2. (a) Schematic diagram and photograph of the THz TDS setup used for experimental measurements. (b) Simulated (solid line) and measured (hollow circles) transmission spectrum of the investigated metamaterial. (c) Amplitude of the transmission of SRRs and CWs in the case of TM polarized incidence plane wave $(E \| \mathrm{CWs})$. (d) Electric field amplitudes and surface current directions when driven by the incident field (denoted by black arrows for greater clarity) from left to right at the lower frequency resonance of $0.36 \mathrm{THz}$, at the transparency peak of $0.46 \mathrm{THz}$, at the higher frequency resonance of $0.74 \mathrm{THz}$ and at the transparency peak of $0.89 \mathrm{THz}$.

CW and SRR are removed, respectively). The result of the investigation is shown in figure 2(c). The following statements can be made. (i) Both electromagnetic spectra of SRRs and CWs exhibit a resonant dip at about $0.41 \mathrm{THz}$ for SRRs and around $0.59 \mathrm{THz}$ for CWs (denoted by arrows), with distinct $Q$ factors of about 1.5 and 9.8 , respectively (the $Q$ factor is obtained from the transmission curve, $Q=\omega_{0} / \Delta \omega$, where $\omega_{0}$ is the resonance frequency and $\Delta \omega$ is the full width at half maximum bandwidth). (ii) Two additional dips appear in the transmission responses of SRRs and CWs (also denoted by arrows). They are weakly pronounced and coincide in frequency around $0.9 \mathrm{THz}$, thus giving rise to the second transmission window at about the same corresponding frequency of $0.9 \mathrm{THz}$.

To gain further understanding of the nature of these resonances, we depicted in figure 2(d) the electric field amplitudes and the surface current distributions at frequencies of $0.36,0.46,0.74$ and $0.89 \mathrm{THz}$. The directions of the currents on the metallic layer are indicated by black arrows for greater clarity. At the resonant frequency of the transmission dip located at $0.36 \mathrm{THz}$, the two symmetric current loops at the right and the left arms of the SRR are parallel to each other and highly radiative. The terahertz field scattered from the metamaterial interferes constructively and gives rise to a dipole-like resonance feature. At the frequency of $0.46 \mathrm{THz}$, 


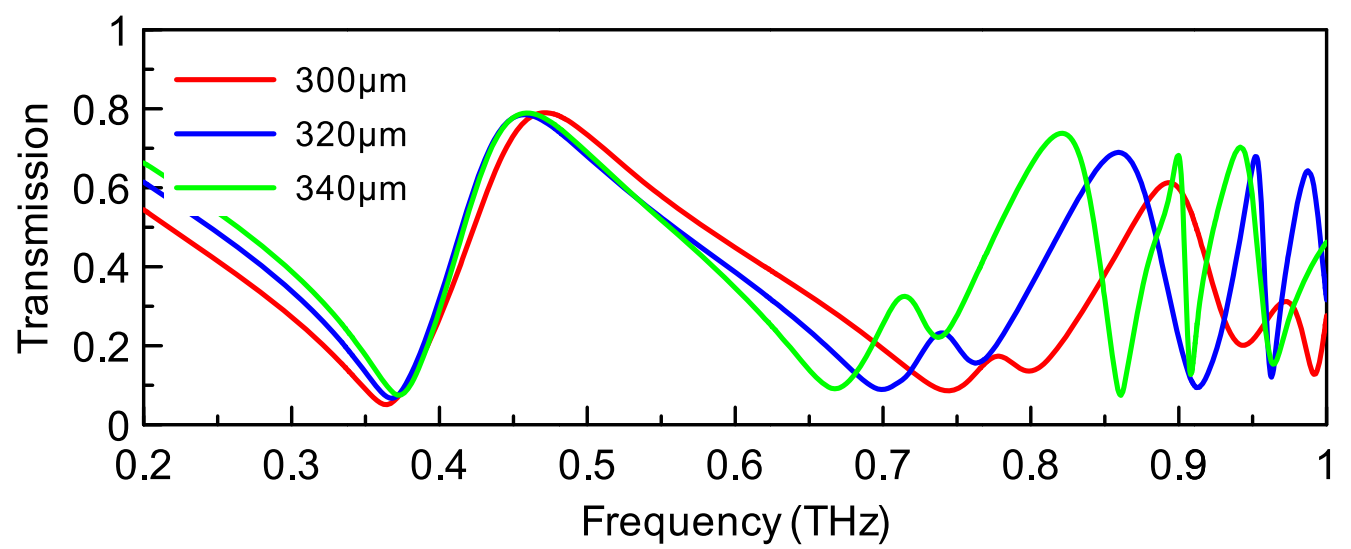

Figure 3. Transmission spectra of the designed metamaterial with different periods $P_{x}=P_{y}$.

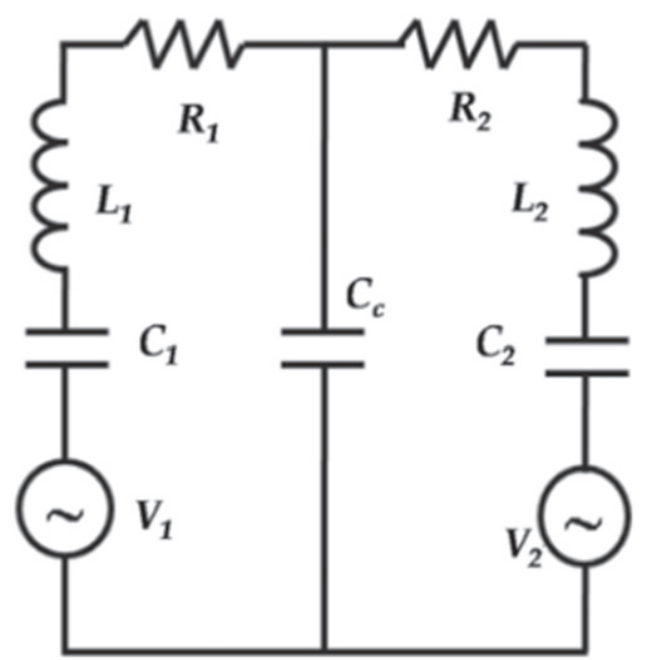

Figure 4. Electric circuit modelling the response of the coupled CW SRR structure.

the structure opens up a transparency window allowing the incident wave to be transmitted with maximum amplitude of about $80 \%$. At the higher frequency resonance of $0.74 \mathrm{THz}$, we observe the excitation of an electric dipole in the $\mathrm{CW}$ and the surface currents of the SRR appear to be suppressed. The currents associated with the transmission peak at $0.89 \mathrm{THz}$ are excited only at the edges of the SRR. One can observe antiparallel current pairs with nodes at the top and bottom of both vertical arms (right and left) of the SRR.

We have further performed simulations on the designed metamaterial by varying the period $P_{x}=P_{y}$ within $300340 \mu \mathrm{m}$ while the dimensions of the SRR and CW were fixed. Figure 3 shows the calculated transmission spectra under different periods $P$. One can observe that the higherorder resonance at $0.74 \mathrm{THz}$ shifts towards lower frequencies as the period $P$ increases, while the changes of the first-order resonance at $0.36 \mathrm{THz}$ is neglected. In short, it is obvious that the first transmission dip at $0.36 \mathrm{THz}$ results from the strong electric resonance in the structure (this is confirmed by the retrieved effective permittivity, but not represented here, which exhibits a highly pronounced Lorentz-like resonance at
$0.36 \mathrm{THz}$ ), while the second transmission dip at $0.74 \mathrm{THz}$ arises in part from interaction of neighboring unit cells.

A possible modelling of the CW SRR structure as two $R L C$ circuits with different lumped elements $R_{1}, L_{1}, C_{1}, R_{2}, L_{2}$ and $C_{2}$, coupled by a common capacitor $C_{\mathrm{c}}$ is given in figure 4 . The left and the right loops of the circuit represent the CW and the SRR, and contain alternating voltage sources to mimic the excitation of the external electric field.

The $R L C$ analysis will be performed by solving equations (1) and (2):

$$
\left(\begin{array}{l}
i_{1} \\
i_{2}
\end{array}\right)=Z^{-1}\left(\begin{array}{l}
v_{1} \\
v_{1}
\end{array}\right)
$$

where $v_{1}$ and $v_{2}$ are the driving voltages; the impedance matrix $Z$ of the circuit is given by

$$
Z=\left(\begin{array}{cc}
-\mathrm{i} \omega L_{1}+R_{1}+\frac{1}{-\omega \mathrm{i} C_{1}} & \frac{1}{-\omega \mathrm{i} C_{\mathrm{c}}} \\
\frac{1}{-\omega \mathrm{i} C_{\mathrm{c}}} & -\mathrm{i} \omega L_{2}+R_{2}+\frac{1}{-\omega \mathrm{i} C_{2}}
\end{array}\right)
$$

The lumped elements will be chosen judiciously by fitting the spectra obtained from the numerical simulations [24]. The topology of the proposed structure offers an extra degree of freedom to gain more insight into the coupling mechanisms between the SRR and the CW, by changing the spatial configuration of the metamolecules. Indeed, we have further carried out calculations with various rotating angles $\theta$ of the $\mathrm{CW}$. Note that the length $l$ of the $\mathrm{CW}$ has been optimized numerically and set to $130 \mu \mathrm{m}$ in order to achieve the angular study. The resonant dip and peak that initially occur at 0.36 and $0.46 \mathrm{THz}$ are slightly shifted to 0.37 and $0.51 \mathrm{THz}$, respectively, as illustrated by the dashed black curve of figure 5(a). As we gradually rotate the $\mathrm{CW}$ resonator, the modulation of the first transparency peak $(0.51 \mathrm{THz})$ and the second transmission broad dip $(0.74 \mathrm{THz})$ are observed. The first transmission dip at about $0.37 \mathrm{THz}$ is not dramatically affected by the rotation of the $\mathrm{CW}$, except for a minor shift towards high frequencies from 0.37 to $0.41 \mathrm{THz}$, which corresponds to about $10 \%$ of tunability (see figure $5(\mathrm{a})$ ).

The first transmission peak around $0.51 \mathrm{THz}$ changes in intensity and splits into two distinct peaks, which move in 

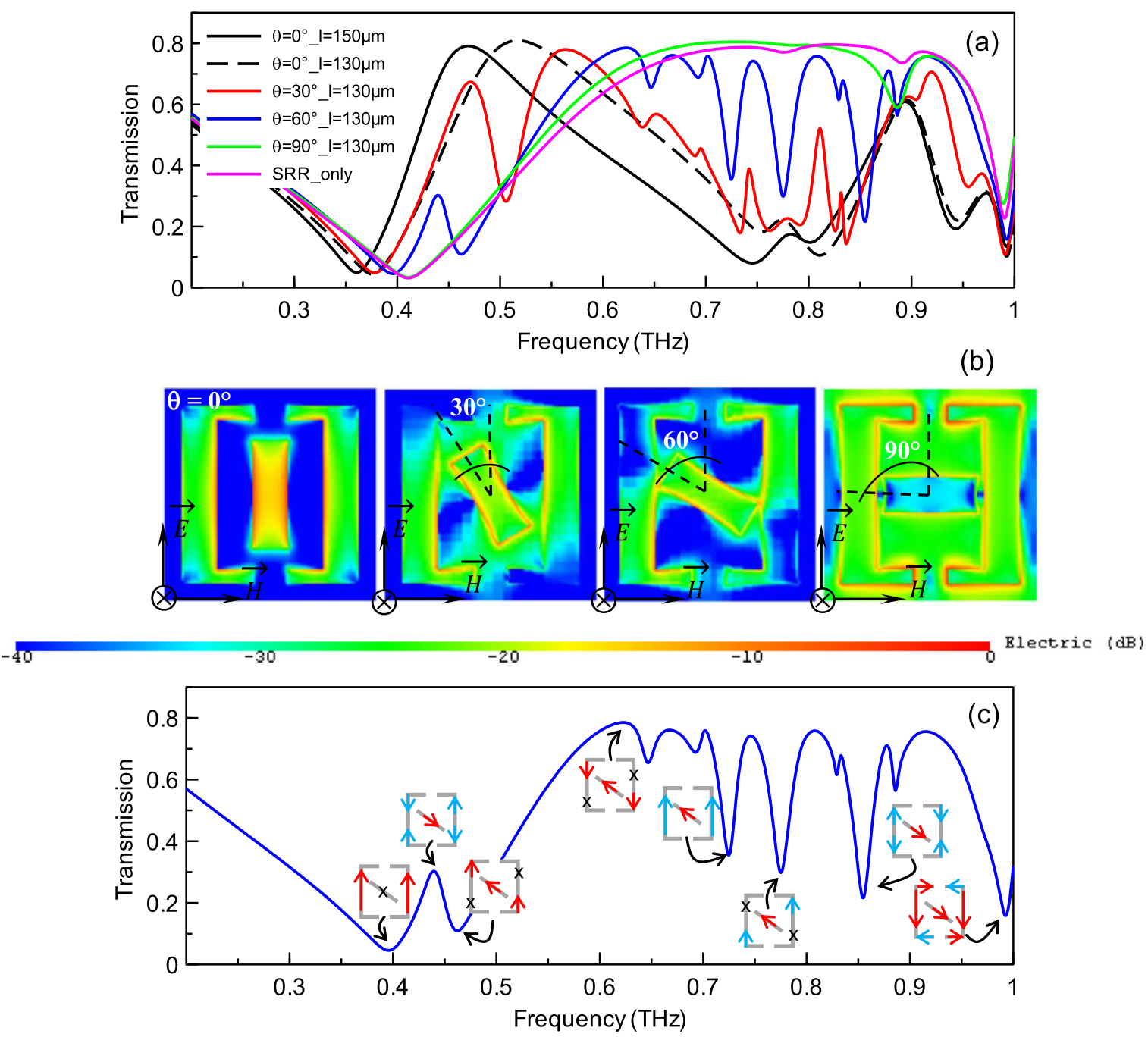

Figure 5. Evolution of the transmission spectra (a) and electric field distribution (b) around the first transmission peak at about $0.51 \mathrm{THz}$ for different rotating angles $\theta$ of the cut wires, with $l=130 \mu \mathrm{m}$. (c) Amplitude of the transmission for the sample with $\theta=60^{\circ}$. The symbols represent the distribution of the surface currents in the SRR and the CW at each transmission dip and peak.

opposite directions (see figure 5(a)). In other words, the transparency window around $0.51 \mathrm{THz}$ tends to disappear; this behavior is accompanied by a gradual transfer of energy from the CW to the SRR (see the spatial distribution of the electric field in figure 5(b) for different rotating angles $\theta$, around the transparency peak at about $0.51 \mathrm{THz}$ ). For the configuration with $\theta=90^{\circ}$, the electromagnetic energy is distributed only on the SRR, which becomes highly radiative. Consequently, the transparency signature disappears and the spectral response of the whole structure is similar to that of the SRR.

The broadband rejection window (i.e., transmission dip at about $0.74 \mathrm{THz}$ ) is also affected by the rotation of the CWs. A series of additional transmission peaks and dips appears within this frequency range, thus translating the higher-order multipolar coupling mechanisms that exist between the SRR and $\mathrm{CW}$ constituents. In order to illustrate these coupling effects, we have depicted in figure 5(c) the amplitude of the transmission for an intermediate case of $\theta=60^{\circ}$. The symbols in figure 5(c) represent the surface currents in the SRR and the $\mathrm{CW}$ at each transmission dip and peak.

For a better understanding of the symbols, the red arrows indicate that the surface currents are strongly excited, while the blue arrows indicate a weak excitation of the surface currents. In contrast, a black cross indicates that the constituents or portions of the metamolecules are not solicited by the incident terahertz wave.

\section{Conclusion}

In conclusion, we have designed, fabricated and experimentally characterized a flexible planar metamaterial operating in the terahertz frequency range. The spectral response of the investigated metamaterial, which exhibits a multiple band frequency response suggests a potential path forward for achieving numerous metamaterials with conformal geometries in the field of antennas, spatial filtering and bio-sensing. Furthermore, the lowering of the symmetry of the unit cell 
induced by the angular study appears to be very promising for polarization conversion applications.

\section{Acknowledgments}

This work was initiated at the University of Limoges. $\mathrm{R}$ Yahiaoui would like to thank MICEL Corporation (www. micel.fr) for providing us with the Kapton ${ }^{\circledR}$ substrate. K Takano, F Miyamaru and M Hangyo were partly supported by a Grant-in-Aid for Scientific Research on Innovative Areas, 'Electromagnetic Metamaterials' (22109003), from the Ministry of Education, Culture, Sports, Science, and Technology (MEXT), Japan.

\section{References}

[1] Fang N, Lee H, Sun C and Zhang X 2005 Science 308534

[2] Schurig D, Mock J J, Justice B J, Cummer S A, Pendry J B, Starr A F and Smith D R 2006 Science 314977

[3] Veselago V G 1968 Sov. Phys. Usp. 10509

[4] Pendry J B, Holden A J, Stewart W J and Youngs I 1996 Phys. Rev. Lett. 764773

[5] Pendry J B, Holden A J, Robbins D J and Stewart W J 1999 IEEE Trans. Microw. Theory Tech. 472075
[6] Shelby R A, Smith D R and Schultz S 2001 Science 29277

[7] Kafesaki M, Tsiapa I, Katsarakis N, Koschny T, Soukoulis C M and Economou E N 2007 Phys. Rev. B 75 235114

[8] Shalaev V M 2007 Nature Photon 141

[9] Valentine J, Zhang S, Zentgraf T, Ulin Avila E, Genov D A, Bartal G and Zhang X 2008 Nature 455376

[10] Rockstuhl C, Menzel C, Paul T, Pertsch T and Lederer F 2008 Phys. Rev. B 78155102

[11] Yahiaoui R, Němec H, Kužel P, Kadlec F, Kadlec C and Mounaix P 2009 Opt. Lett. 343541

[12] Miyamaru F, Kuboda S, Taima K, Takano K, Hangyo M and Takeda M W 2010 Appl. Phys. Lett. 96081105

[13] Hangyo M, Tani M and Nagashima T 2005 Int. J. Infrared Millimeter Waves 261661

[14] Tuniz A et al 2013 Nat. Commun. 42706

[15] Gaillot D P, Croënne C and Lippens D 2008 Opt. Express 163986

[16] Grant J, Ma Y, Saha S, Khalid A and Cumming D R S Opt. Lett. 363476

[17] Yahiaoui R, Guillet J P, de Miollis F and Mounaix P 2013 Opt. Lett. 384988

[18] Cong L et al 2013 Appl. Phys. Lett. 103171107

[19] Grady N K et al 2013 Science 3401304

[20] Yoshida H et al 2007 Appl. Phys. Lett. 91253901

[21] Debus C and Bolivar P H 2007 Appl. Phys. Lett. 91184102

[22] Tao H et al 2011 Adv. Mater. 233197

[23] Miyamaru F et al 2014 J. Infrared Milli Terahz Waves 35198

[24] Meyrath T P, Zentgraf T and Giessen H 2007 Phys. Rev. B 75 205102 